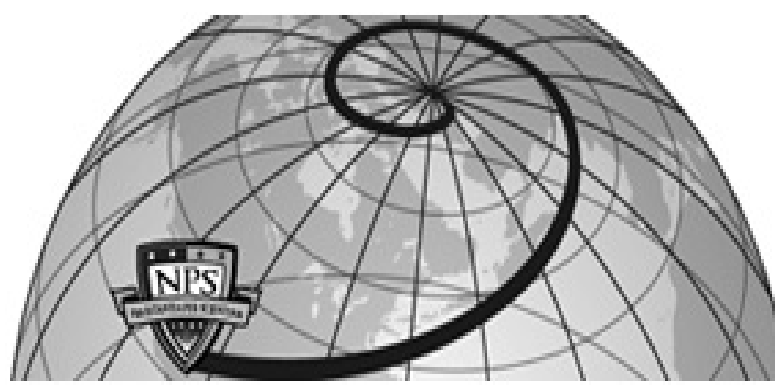

Calhoun: The NPS Institutional Archive DSpace Repository

\title{
An integrated GPS/INS navigation system for small AUVs using an asynchronous Kalman filter
}

Yun, X.; Hernandez, G. C.; Bachmann, E.R.; Mcghee, R.B.; Healey, A.J.

https://hdl.handle.net/10945/41287

This publication is a work of the U.S. Government as defined in Title 17, United States Code, Section 101. Copyright protection is not available for this work in the United States.

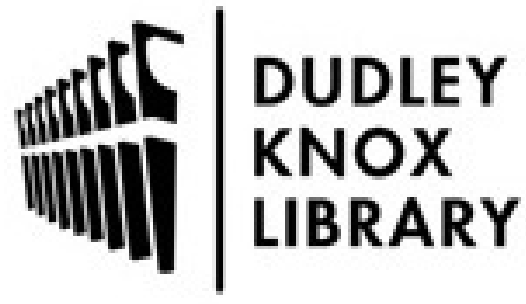

http://www.nps.edu/library
Calhoun is the Naval Postgraduate School's public access digital repository for research materials and institutional publications created by the NPS community. Calhoun is named for Professor of Mathematics Guy K. Calhoun, NPS's first appointed -- and published -- scholarly author.

Dudley Knox Library / Naval Postgraduate School 411 Dyer Road / 1 University Circle Monterey, California USA 93943 


\title{
An Integrated GPS/INS Navigation System for Small AUVs Using an Asynchronous Kalman Filter
}

\author{
X. Yun, G.C. Hernandez, E.R.Bachmann, R.B.McGhee and A.J. Healey \\ Naval Postgraduate School \\ Monterey, CA 93943 \\ yun@nps.navy.mil
}

\begin{abstract}
A Small AUV Navigation System (SANS) is being developed at the Naval Postgraduate School. The SANS is an integrated GPS/INS navigation system composed of low-cost, small-size components. It is designed to demonstrate the feasibility of using a low-cost Inertial Measurement Unit (IMU) to navigate between intermittent GPS fixes. This paper reports recent improvements to the SANS hardware, latest testing results after compensating heading-dependent derivations in the TCM-2 compass measurements, and development of an asynchronous Kalman filter for improved position estimation.
\end{abstract}

Keywords: INS, GPS, AUV, Navigation, Kalman Filter.

\section{Introduction}

Autonomous Underwater Vehicles (AUVs) are capable of a variety of overt and clandestine missions. Such vehicles have been used for inspection, mine counter measures, survey and observation [1]. Accurate navigation is a crucial aspect of each of these missions. The Global Positioning System (GPS) is capable of providing this accuracy if integrated with an Inertial Navigation System (INS) to compensate for intermittent reception caused by either wave action or deliberate submergence.

A Small AUV Navigation System (SANS) is being developed to demonstrate the feasibility of using low-cost and small-size Inertial Measurement Unit (IMU) to navigate between DGPS fixes. The goal is a self-contained, internally or externally mountable package which can be easily carried by a small AUV. The initial installation target for the SANS is the Naval Postgraduate School "Phoenix" AUV [2, 3]. The first prototype of

\footnotetext{
"To whom all correspondence should be addressed Correspondence address: Department of Electrical and Computer Engineering, Code EC/Yx, Naval Postgraduate School, Monterey, CA 93943-5121.
}

the SANS system (called SANS I) was separated into two subsystems $[4,5]$. The IMU, water speed sensor, compass, GPS antenna, and data logging computer were housed in one package and placed in a towfish. The GPS receiver, DGPS antenna, and data processing computer were in the towing vessel. The data collected by the towfish subsystem were transmitted to the processing computer through a modem cable.

The second generation of the SANS system, or SANS II, was totally contained in a single package. The software of SANS I and SANS II was based on a twelve-state complementary filter with constant gains. The value of these gains was initially selected based on bandwidth considerations, and later tuned based on results from bench testing and ground vehicle testing. SANS II was the subject of extensive tilt table testing, bench testing, and ground vehicle testing. Early testing results were reported in [6]. The latest testing results of SANS II are described in Section 2 of this paper. Tilt table tests showed that attitude sensing was achieved to an accuracy of one degree or better under the most demanding of circumstances [7]. This is in agreement with the findings of other investigations [8]. Bench testing was conducted to properly tune the filter gains. Ground vehicle testing, where the SANS system was mounted on a moving golf cart, was also conducted to demonstrate the feasibility of the SANS system.

SANS III is the current version of the system. This paper reports recent improvements to the SANS hardware and software. The 486based ESP computer used in the previous versions of SANS is now replaced by an AMD 586DX133 based PC/104 computer to provide more computing power and, more importantly, to increase reliability and compatibility by using PC/104 industrial standards [9].

In the current version of SANS, the position and velocity estimation part of the constant-gain filter used in SANS I and SANS II is replaced by an asynchronous Kalman filter. The new fil-

0-7803-5190-8/98/\$10.00 @1998 IEEE. 


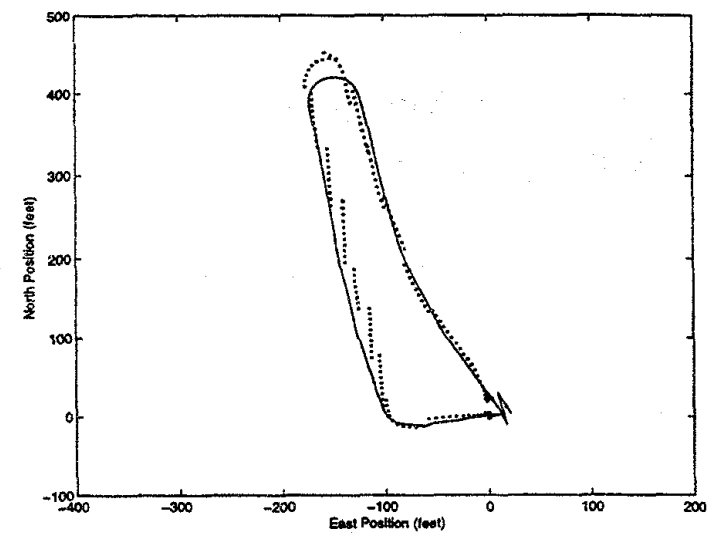

Figure 1: Position plot for ground vehicle testing with DGPS update every 10 seconds and prior to compass calibration (dotted line); continuous DGPS position (solid line).

ter has six states for orientation estimation (still constant gain), and eight states for position estimation. Low-frequency DGPS noise is explicitly modeled based on an experimentally obtained autocorrelation function. Ocean currents are also modeled as a low-frequency random process. Finally, the asynchronous nature of DGPS measurements resulting from AUV submergence or wave splash on the DGPS antennas is also taken into account by adopting an asynchronous Kalman filter as the basis for the SANS position estimation software. Matlab simulation studies of the asynchronous filter have been conducted, and simulation results are presented.

\section{Testing Results of SANS II}

Early testing results of SANS II were reported in [6]. This section presents findings resulting from compass calibration, and testing results of SANS II after compensating heading-dependent errors in compass measurements.

The SANS uses a TCM-2 digital compass to obtain low-frequency heading information. A detailed investigation into compass calibration reveals that, in our test environment, the TCM-2 compass has heading-dependent deviations of up to five degrees. With the aid of a precision transit, a deviation table was established and utilized to compensate heading-dependent deviations in the software. After compensating compass deviations, results from ground vehicle testing (with the previous constant-gain filter) indicate that the SANS system is able to navigate with 10 meter accuracy for more than three minutes between DGPS fixes.

\subsection{Compass Calibration}

To obtain heading information in SANS, the angular rate sensor is used as a high frequency input source and the digital compass (Precision Navigation Electronic Compass, Model TCM2) is used as low frequency data source. In a series of ground vehicle tests [6], it was noted that the SANS heading is usually off by a few degrees. Figure 1 shows a typical run from ground vehicle testing with DGPS updates every 10 seconds. The solid line is the filtered vehicle track with continuous DGPS data, and is taken as a reference. The dotted line is the filtered vehicle track when DGPS information is provided to the filter every 10 seconds. The vehicle starts from $(0,0)$ coordinate, moves westward, continues northward after a right turn, and returns to the starting location after making a Uturn. It is evident, particularly from the northerly run, that the filter is not receiving accurate directional information. A comparison of raw compass data and the filtered heading points to the compass as the source of heading deviation.

The investigation of compass derivation was focused on three areas: possible interference produced by the golf cart electric motor, vibration, and the deviation of the compass itself. The TCM2 compass has a self calibration routine which is designed to remove the effects of static magnetic fields caused by ferrous materials in the vicinity of the compass. The calibration routine is not capable of compensating for dynamic magnetic field distortions like those caused by an electric motor. To find the effect of the electric motor on the compass, a series of tests were conducted.

The tests were performed by jacking up the rear wheels of the golf cart so that they could spin freely while the motor was on. The motors were turned on for 30 second intervals followed by a 30 second off period. During the off time, the wheels continued to rotate for approximately 10-15 seconds until they came to a stop. This test allowed changes in heading due to motor engagement to be observed. The same test was performed four times rotating the cart through the cardinal points. The result of one such test (with the vehicle facing east) is depicted in Figure 2. Testing results indicate that there is interference, but its magnitude appears to be limited to approximately a half of a degree. The figure also indicates that the noise is at a relatively high frequency which can be compensated for with an appropriate value of the filter gain. Similar results were obtained with a full load applied to the motor.

The effect of vibration was tested by slightly tapping the board (where the compass is mounted) with a screwdriver and a finger. Figure 3 shows 


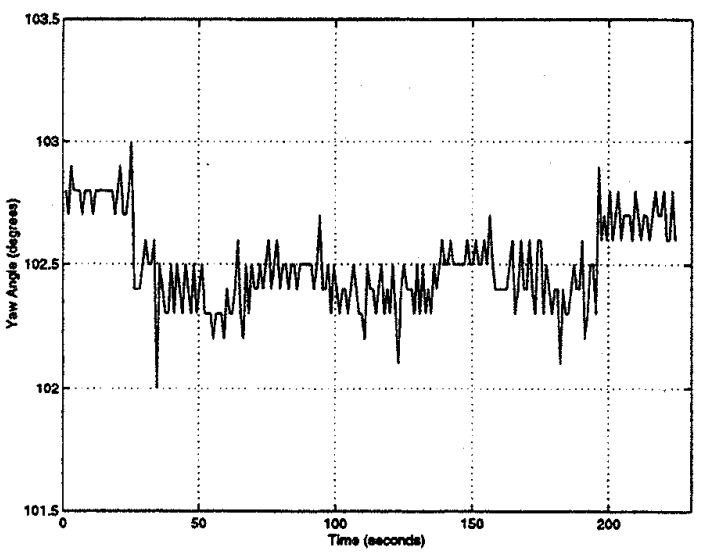

Figure 2: Effect of the golf cart motor on compass measurements.

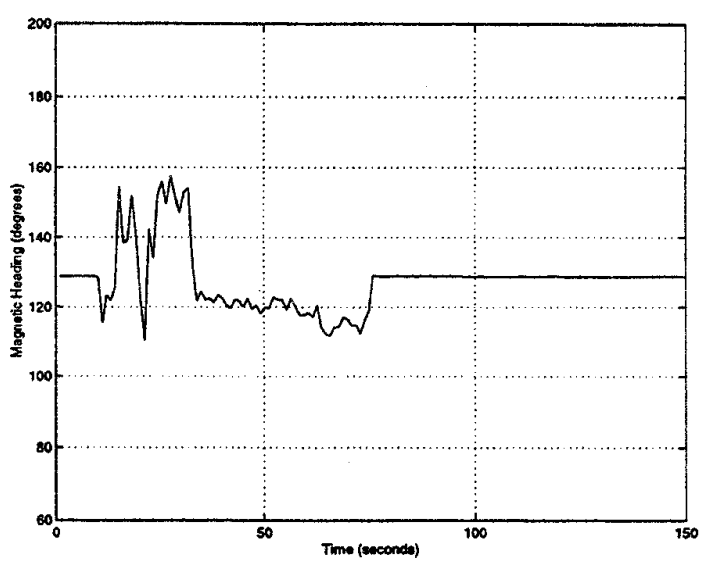

Figure 3: Effect of vibration on compass measurements.

compass reading when subjected to vibration. It is seen that vibration clearly plays a much greater role in compass error than the electric motor. Again, these deviations are mostly in high frequency range and will be filtered out with an appropriate filter gain.

In ground vehicle testing, it was noticed that heading error during east-west runs was not as large as during north-south runs. A final test was conducted to determine, if any, compass deviations that are heading dependent exist. The test was performed by swinging the compass and comparing the compass reading with a reference. The instrument used as the reference was a transit manufactured by W. and L.E. Gurley with an accuracy of 0.5 degrees. It has a calibrated, balanced magnetic compass mounted within its body. By mounting the transit in line with the TCM-

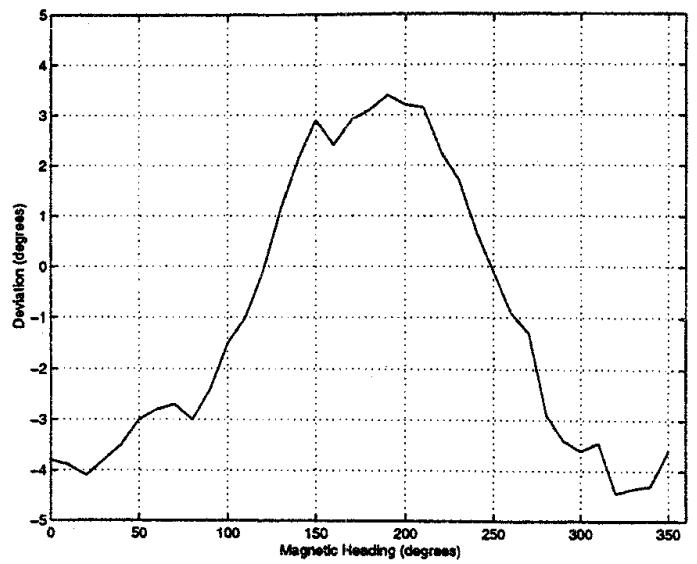

Figure 4: TCM-2 compass deviation.

2 compass, a comparison is made between the two indicated headings. The compass was swung through the entire 360 degrees, taking measurements every 10 degrees. Figure 4 shows the difference between measurements of the transit and those of the TCM-2 compass. Figure 4 is qualitatively in agreement with the observation made during ground vehicle testing where north-south runs show greater heading errors than east-west runs. Using this data, a table lookup and linear interpolating function were added to the SANS filter code to compensate the heading dependent derivation.

\subsection{Ground Vehicle Testing}

After compensating heading-dependent derivations in compass measurements, similar ground vehicle testing as reported in [6] was conducted. In ground vehicle testing, the SANS system was installed on a golf cart. The cart was driven in a parking lot. The ground vehicle tests described in [6] were conducted without direct measurement of vehicle speed. The vehicle speed was crudely estimated based on the length of the traveling course and traveling time, and assumed a constant value throughout testing. To better estimate the vehicle speed, a bicycle wheel was instrumented and trailed behind the golf cart. The bicycle wheel was provided with a reed switch and a magnet that generates a pulse upon every revolution. Counting the elapsed time between two pulses provides a better estimate of the average speed [10].

After implementing the bicycle wheel speed measurement and compensating heading-dependent compass derivation (based on Figure 4), another ground vehicle test; was conducted. The result of this test is depicted in Figure 5. The dotted 


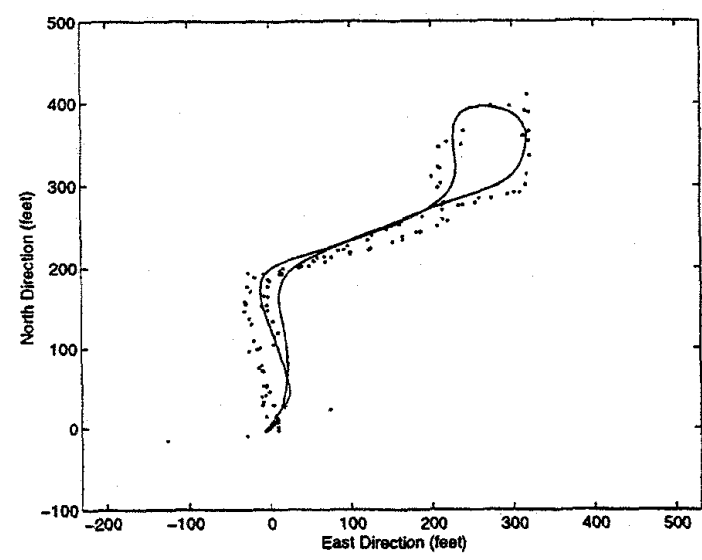

Figure 5: Ground vehicle test result with calibrated compass and bicycle wheel speed measurement. Dotted line: with DGPS; solid line: without DGPS.

line represents the vehicle trajectory with continuous DGPS data. The solid line is the trajectory of the vehicle following the same path without DGPS data during the entire run of 3.2 minutes. Taking the dotted line as the reference, it is seen that the result of dead reckoning navigation without DGPS is accurate to within 10 meters or less.

\section{Development of SANS III}

\subsection{Improvements in Hardware}

In the first two versions of SANS, a 386- or 486-based ESP computer was utilized as the main computing power for data acquisition and processing. In the past several years, PC/104 has become the industrial standard for embedded computing applications. In SANS III, an AMD 586DX133 PC/104 module from Real Time Devices [11] is adopted for data acquisition and processing (see Figure 6). It is loaded with the MS-DOS operating system. This processor provides more computing power, which in turn permits a faster sampling rate. In addition, use of the $\mathrm{PC} / 104$ industrial standard enhances the reliability, flexibility, and compatibility of the SANS system. The PC/104 CPU module also has a $12 \mathrm{MB}$ disk on a chip that will store the SANS executable codes.

Two serial ports usually come standard on PCs. For the present application, however, four serial ports are needed for connecting DGPS, compass, IMU, and water speed sensor. A four serial port PC/104 module from Sealevel Systems, Inc. was acquired for this purpose [12].

The Systron Donner MotionPak IMU is now replaced by a Crossbow DMU-VG six-axis IMU [13].

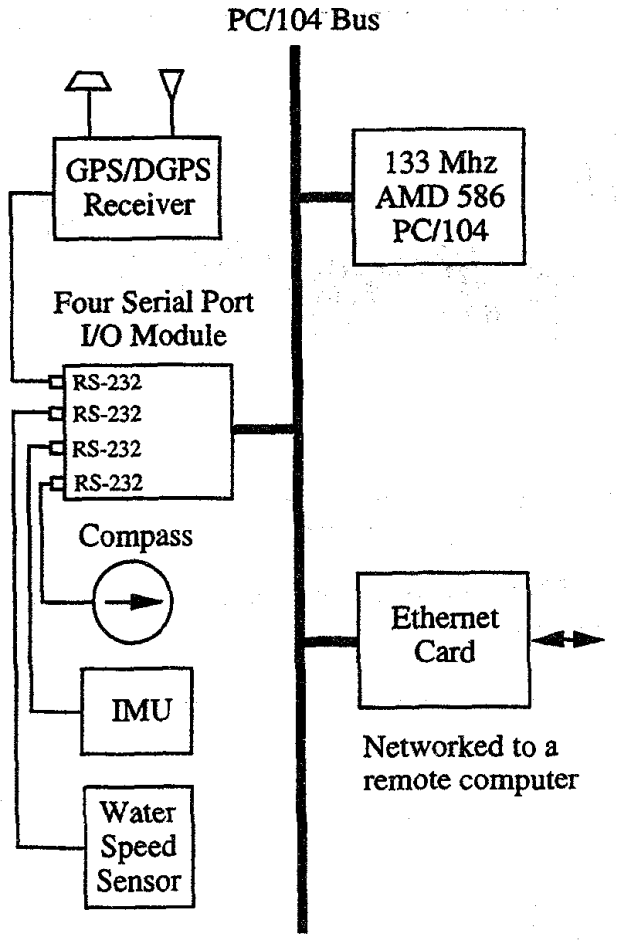

Figure 6: SANS III hardware configuration.

The two units are about the same size, but the Crossbow unit has an internal $A / D$ board, delivering data in RS-232 format. (Analog data is also available if it is set in a different output mode.) This eliminates the need for a PC/104 A/D module, and therefore reduces the overall size of the SANS system. Furthermore, the Crossbow unit consumes significantly less power.

\subsection{Asynchronous Kalman Filter}

The navigation software in the previous versions of SANS was based on a twelve-state constant-gain filter. Filter gains were initially selected based on bandwidth considerations and later tuned based on tilt table and bench testing. This filter is being replaced by an asynchronous Kalman filter in SANS III. This filter has six states for orientation estimation (still constant gain), and eight states for position estimation. The part of the filter for orientation estimation remains the same as reported in [6] and will not be repeated here. The process model for position estimation is depicted in Figure 7 . In this model, the velocity relative to water, ocean current, and GPS bias (state variables $x_{1}$ through $x_{6}$ ) are modeled as colored signals generated by white noises $q_{1}, \ldots, q_{6}$ through first-order systems. The 


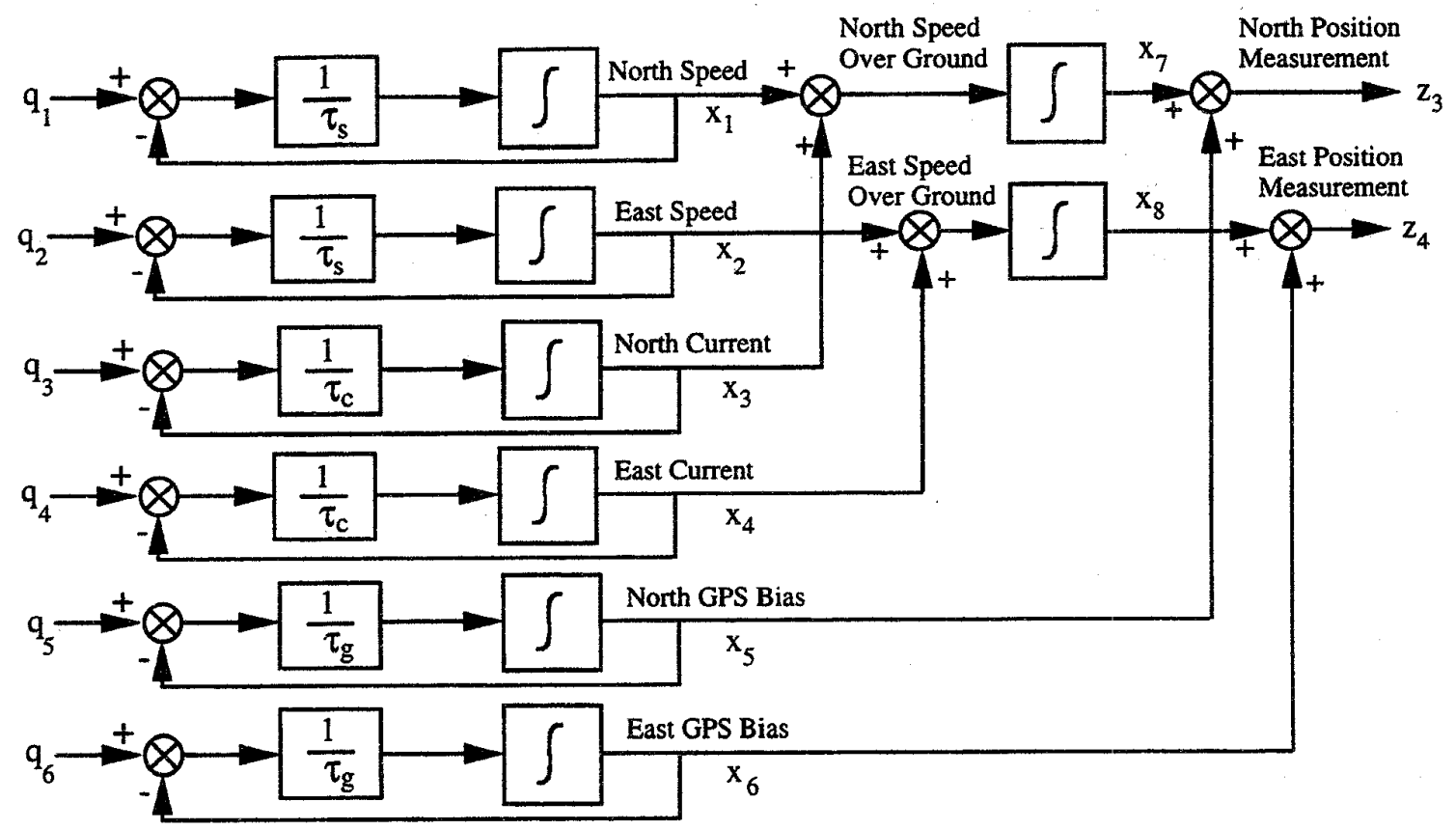

Figure 7: Process model of the Kalman filter.

time constant $\tau_{s}$ and $\tau_{g}$ are on the order of minutes, and $\tau_{c}$ is on the order of hours The velocity relative to the ground is obtained by summing the velocity relative to water $\left(x_{1}\right.$ and $\left.x_{2}\right)$ and the ocean current $\left(x_{3}\right.$ and $\left.x_{4}\right)$, and integrated to obtain position estimation ( $x_{7}$ and $\left.x_{8}\right)$. Therefore, the Kalman filter state equations are characterized by:

$$
\begin{aligned}
\dot{x}_{1} & =-\frac{1}{\tau_{s}} x_{1}+\frac{1}{\tau_{s}} q_{1} \\
\dot{x}_{2} & =-\frac{1}{\tau_{s}} x_{2}+\frac{1}{\tau_{s}} q_{2} \\
\dot{x}_{3} & =-\frac{1}{\tau_{c}} x_{3}+\frac{1}{\tau_{c}} q_{3} \\
\dot{x}_{4} & =-\frac{1}{\tau_{c}} x_{4}+\frac{1}{\tau_{c}} q_{4} \\
\dot{x}_{5} & =-\frac{1}{\tau_{g}} x_{5}+\frac{1}{\tau_{g}} q_{5} \\
\dot{x}_{6} & =-\frac{1}{\tau_{g}} x_{6}+\frac{1}{\tau_{g}} q_{6} \\
\dot{x}_{7} & =x_{1}+x_{3} \\
\dot{x}_{8} & =x_{2}+x_{4}
\end{aligned}
$$

Acceleration signals from the attitude estimation part of the SANS III filter were not used for velocity estimation because they were judged to be too noisy to provide useful information in comparison to values for velocity obtained directly from an accurate water speed sensor. With the process model shown in Figure 7, the measurements used for position estimation are the velocity relative to water provided by the water speed sensor and position information provided by DGPS. The velocity measurements are synchronous and available at every sampling time. DGPS information is asynchronous and is only available when the AUV is surfaced. The two synchronous measurement equations are:

$$
\begin{aligned}
& z_{1}=x_{1}+v_{1} \\
& z_{2}=x_{2}+v_{2}
\end{aligned}
$$

where $v_{i}, i=1,2$ are white noise. That is, it is assumed that the velocity measurements contain additive white noise. The two asynchronous measurement equations are:

$$
\begin{aligned}
& z_{3}=x_{7}+x_{5} \\
& z_{4}=x_{8}+x_{6}
\end{aligned}
$$

Because of asynchronous nature of DGPS measurements, an asynchronous Kalman filter is needed to provide position estimation. The design of the filter follows the standard procedures [14], with one notable difference. At the time when GPS measurements are available, the vector of measurements is four dimensional. At any other times, it is two dimensional. Consequently the dimension of the Kalman filter gain matrix $K$ 


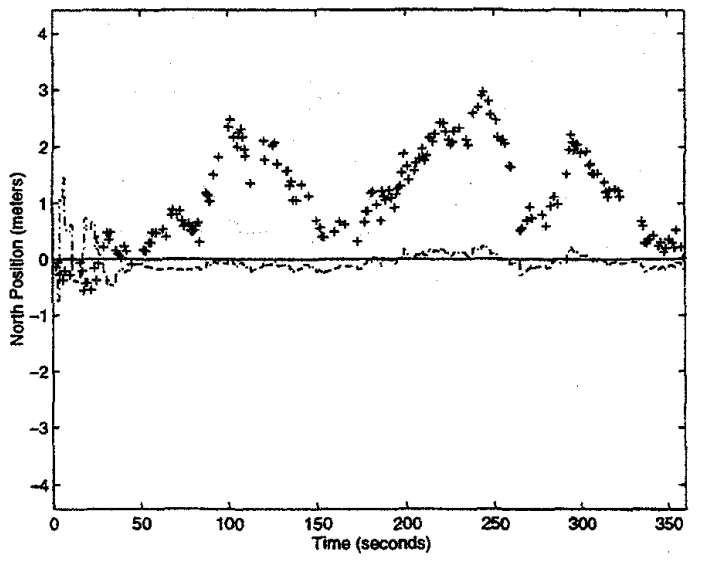

Figure 8: North position vs. time. Solid line: actual position (zero); dash line: estimated position; plus sign: DGPS measurement.

is either $8 \times 4$ or $8 \times 2$, depending on the dimension of measurements. It is noted that the dimension of states is 8 .

\subsection{Simulation Results}

Prior to implementing the asynchronous Kalman filter in SANS III, it was first simulated using Matlab. Simulation results are presented in this subsection. Asynchronism is coded as follows. At a synchronous sampling rate of $100 \mathrm{~Hz}$, the filter takes measurements of water speed and updates the position estimation. DGPS signals are made available at a rate of about $1 \mathrm{~Hz}$. As soon as DGPS signals are received, they will be utilized, along with water speed measurements, to update the position estimation at the next (synchronous) sample time.

Simulation results are shown in Figures 8 through 10. Actual north and east position are zero in all cases. Figure 8 shows the estimated north position (dash line) along with DGPS measurements (plus sign). After 50 seconds, the estimated north position remains within one meter from the actual value. Figure 9 shows the estimated east position and DGPS measurements. It is seen that initial estimation error is relatively large, but the filter finally converges. Estimated north position versus estimated east position is ploted in Figure 10.

\section{Current and Future Work}

The current effort is on hardware integration and software development of SANS III. All the hardware components shown in Figure 6 have been

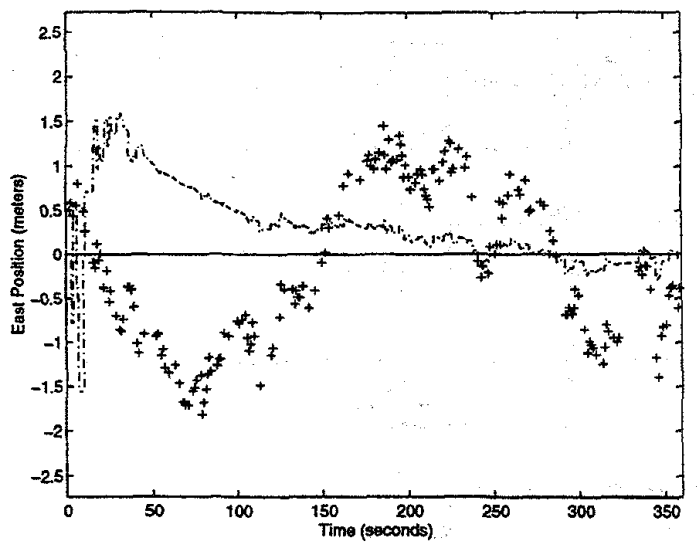

Figure 9: East position vs. time. Solid line: actual position (zero); dash line: estimated position; plus sign: DGPS measurement.

acquired or are already available from the previous version. The new hardware configuration is under testing and evaluation. The navigation software of SANS III will be based on the asynchronous Kalman filter discussed in Section 3, and is currently under development. When completed, SANS III will be tested at sea in Monterey Bay using the Phoenix AUV.

A parallel effort is proceeding on tracking of human body motion using inertial sensors. Three angular rate sensors, three accelerometers, and a three-axis magnetometer are being packaged into a 1.25 inch cube to form an integrated orientation sensor. Multiple sensors are to be placed on human limbs. These sensors allow determination of orientation of each limb segment and thus reconstruction of body posture. Inertial tracking will eliminate the restricted range limitations suffered by most other body tracking technologies.

Euler angles are used to represent orientation in the SANS filter. The use of Euler angles is adequate for AUV navigation, but it does not allow to motion tracking through the vertical as would be necessary for body tracking. An orientation filter based on quaternions is currently being developed.

\section{Conclusion}

This paper is a progress report concerning the SANS system development. The latest testing results of SANS II are described. These results demonstrate that the SANS is able to navigate with 10 meter accuracy for more than three minutes between DGPS fixes. To further reduce size and improve navigation accuracy of the SANS system, a new version (SANS III) is being developed. 


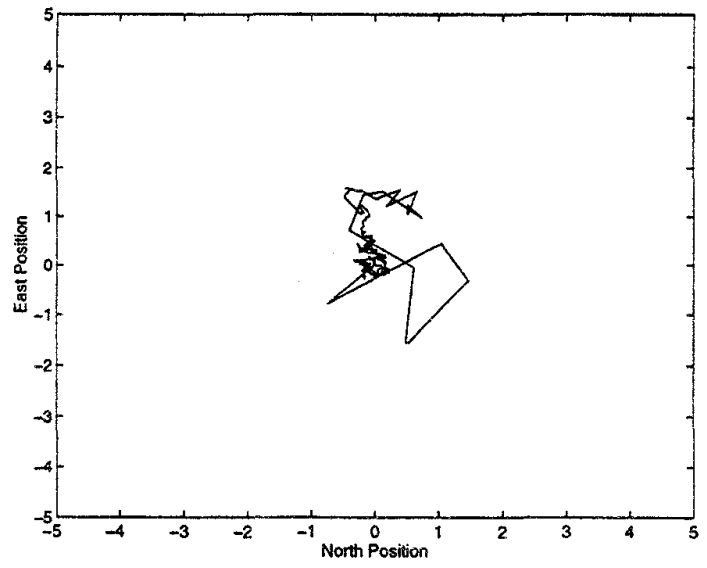

Figure 10: Estimated north position vs. estimated east position.

Improvements to the SANS hardware and software are described. The new hardware configuration uses a PC/104 computer and a Crossbow IMU. The new software is based on an asynchronous Kalman filter. Matlab simulation results of the asynchronous Kalman filter are also presented.

\section{Acknowledgment}

This research was supported in part by $\mathrm{Na}$ tional Science Foundation under grant No. CDA9729814, and by Florida Atlantic University under Contract No. 611588 .

\section{References}

[1] J. Yuh. Underwater Robotic Vehicles: Design and Control. TSI Press, 1995.

[2] D.B. Marco, A.J. Healey, and R.B. McGhee. Autonomous underwater vehicle: Hybrid control of mission and motion. Autonomous Robots, (3):169-186, 1996.

[3] Don Brutzman, Tony Healey, Dave Marco, and Bob McGhee. The Phoenix autonomous underwater vehicle. In D. Kortenkamp, R.P. Bonasso, and R. Murphy, editors, Artificial Intelligence and Mobile Robots: Case studies of successful robot systems, chapter 13, pages 323-360. MIT Press, Cambridge, MA, March 1988.

[4] R.B. McGhee et al. An experimental study of an integrated GPS/INS system for shallowwater AUV navigation (SANS). In Proceedings of 9th International Symposium on Un- manned Untethered Submersible Technology (UUST), Durham, NH, September 1995.

[5] E.R. Bachmann, et al. Evaluation of an integrated GPS/INS system for shallow water AUV naviation. In 1996 IEEE Symposium on Autonomous Underwater Vehicle Technology, pages 268-275, Monterey, CA, June 1996.

[6] X. Yun, E.R. Bachmann, R.B. McGhee, R.H. Whalen, R.L. Roberts, R.G. Knapp, A.J. Healey, and M.J. Zyda. Testing and evaluation of an integrated GPS/INS system for small auv navigation (sans). In Proceedings of the 10th International Symposium on Unmanned Untethered Submersible Technology (UUST), Durham, NH, September 1997.

[7] R.L. Roberts. Implementation and evaluation of an integrated self-contained GPS/INS shallow-water AUV navigation system (SANS). Master's thesis, Naval Postgraduate School, Monterey, California, March 1997.

[8] R. Wolf, G.W. Hein, B. Eissfeller, and E. Loehnert. An integrated low cost GPS/INS attitude determination and position location system. In Proceedings of ION GPS-96 Meeting, pages 975-981, Kansas City, MO, September 1996.

[9] Glenn C. Hernandez. An integrated INS/GPS navigation system for small AUV using an asynchronous Kalman filter. Master's thesis, Naval Postgraduate School, Monterey, California, June 1998.

[10] Randall Knapp. Calibration and evaluation of a water speed indicator and compass for the small autonomous underwater vehicle navigation filter. Master's thesis, Naval Postgraduate School, Monterey, California, December 1997.

[11] CMV586DX133 CPU module user's manual. Technical report, Real Time Devices, Inc., September 1997.

[12] C4-104 user manual. Technical report, Sealevel Systems, Inc., January 1997.

[13] Crossbow six axis dynamic measurement unit specification. Technical report, Crossbow, San Jose, CA, January 1998.

[14] Robert Grover Brown and Patrick Y.C. Hwang. Introduction to Random Signals and Applied Kalman Filtering. John Wiley \& Sons, New York, NY, third edition, 1997. 